\title{
A criterion for the existence of a plane model with two inner Galois points for algebraic curves
}

\author{
Kazuki Higashine
}

(Received October 2, 2020)

(Revised January 18, 2021)

\begin{abstract}
A criterion for the existence of a plane model with two non-smooth Galois points for algebraic curves is presented, which is a generalization of Fukasawa's criterion for two smooth Galois points. Multiplicities and order sequences at Galois points are also described in detail.
\end{abstract}

\section{Introduction}

Let $k$ be an algebraically closed field of characteristic $p \geq 0$, and let $C \subset \mathbb{P}^{2}$ be an irreducible (possibly singular) plane curve of degree $d \geq 4$ over $k$. For points $P, Q \in \mathbb{P}^{2}$ with $P \neq Q$, the line passing through $P, Q$ is denoted by $\overline{P Q}$. We consider the projection $\pi_{P}: C \rightarrow \mathbb{P}^{1} ; Q \mapsto \overline{P Q}$ with the center $P \in \mathbb{P}^{2}$. If the field extension $k(C) / \pi_{P}^{*} k\left(\mathbb{P}^{1}\right)$ of function fields induced by $\pi_{P}$ is Galois, then $P$ is called a Galois point for $C$. This notion was introduced in 1996 by Yoshihara (see $[3,13,16]$ ). For a Galois point $P$, the associated Galois group $G_{P}=\operatorname{Gal}\left(k(C) / \pi_{P}^{*} k\left(\mathbb{P}^{1}\right)\right)$ is called a Galois group at $P$. Furthermore, a Galois point $P$ is called a smooth Galois point (resp. a non-smooth Galois point, an inner Galois point, an outer Galois point) if $P$ is a smooth point of $C$ (resp. a singular point of $C$, a point contained in $C$, a point not contained in $C$ ), after $[11,12,15]$. Note that the definition of an inner Galois point in [5] is equivalent to the definition of a smooth Galois point in this article.

In 2016, Fukasawa [5] presented a criterion for the existence of a birational embedding of a smooth projective curve into a projective plane with two smooth Galois points and obtained new examples of plane curves with two smooth Galois points by using this criterion. On the other hand, there have been some known examples of plane curves with two or more non-smooth Galois points. For example, the Ballico-Hefez curve ([4, Theorem 1]), some self-dual curves ([9, Theorem 17]), the (plane model of) Giulietti-Korchmáros

The author was partially supported by Grant-in-Aid for JSPS Research Fellows (No. 20J12384). 2010 Mathematics Subject Classification. 14H05, 14H37, 14H50.

Key words and phrases. automorphism group, Galois group, Galois point, plane curve. 
curve ([8, Theorem 2]), the $\left(q^{3}, q^{2}\right)$-Frobenius nonclassical curve ([1, Theorem 1]), and the Artin-Schreier-Mumford curve (proof of [6, Theorem 1]) are such curves. However, these examples are not intended to actively focus on nonsmooth Galois points. Only few research studies have focused on non-smooth Galois points. Takahashi [15] studied plane quintic curves with a double point $P$ and determined defining equations when $P$ is a Galois point. As far as the author knows, this is the only study that focused on a non-smooth Galois point so far. In order to study non-smooth Galois points systematically, it is good to have a criterion for non-smooth Galois points.

In this article, we extend Fukasawa's criterion [5, Theorem 1] to all cases with two (possibly non-smooth) Galois points. Let $X$ be a (reduced, irreducible) smooth projective curve over $k$, and let $k(X)$ be its function field. The full automorphism group of $X$ is denoted by $\operatorname{Aut}(X)$. For a finite subgroup $G \subset \operatorname{Aut}(X)$ and a point $P \in X$, the stabilizer of $P$ in $G$ (resp. the orbit of $P$ under $G$ ) is denoted by $G(P)$ (resp. $G \cdot P$ ). Furthermore, the quotient curve of $X$ by $G$, that is, the smooth projective curve corresponding to the fixed field of $k(X)$ by $G$, is denoted by $X / G$. The following are our main theorems.

TheOrem 1. Let $G_{1}$ and $G_{2}$ be finite subgroups of $\operatorname{Aut}(X)$ and let $P_{1}$ and $P_{2}$ be different points of $X$. Then there exists a birational embedding $\varphi: X \rightarrow$ $\mathbb{P}^{2}$ such that $\varphi\left(P_{1}\right)$ and $\varphi\left(P_{2}\right)$ are different inner Galois points, that $G_{\varphi\left(P_{i}\right)}=G_{i}$ for $i=1,2$, and that $L=\overline{\varphi\left(P_{1}\right) \varphi\left(P_{2}\right)}$ is not a tangent line at $\varphi\left(P_{1}\right)$, if and only if the following conditions are satisfied:

(a) $X / G_{1} \cong \mathbb{P}^{1}, X / G_{2} \cong \mathbb{P}^{1}$,

(b) $G_{1} \cap G_{2}=\{1\}$, and

(c) one of the following holds:

(c- i ) $P_{1} \notin G_{1} \cdot P_{2}, P_{2} \notin G_{2} \cdot P_{1}, G_{1} \cdot P_{2} \cap G_{2} \cdot P_{1} \neq \varnothing$, and $\left|G_{1}\left(P_{2}\right)\right|$ $=\left|G_{2}\left(P_{1}\right)\right|$.

(c-ii) $G_{1} \cdot P_{2} \cap G_{2} \cdot P_{1}=\varnothing$.

(c-iii) $P_{1} \notin G_{1} \cdot P_{2}, G_{1} \cdot P_{2} \cap G_{2} \cdot P_{1} \neq \varnothing$ and $\left|G_{1}\left(P_{2}\right)\right|>\left|G_{2}\left(P_{1}\right)\right|$.

Furthermore, for any $\varphi$ as in the above, the following hold:

(i ) $L$ is not a tangent line at $\varphi\left(P_{2}\right)$ with $L \cap \varphi(X) \supsetneq\left\{\varphi\left(P_{1}\right), \varphi\left(P_{2}\right)\right\}$ if and only if condition (c-i) is satisfied.

(ii) $L$ is not a tangent line at $\varphi\left(P_{2}\right)$ with $L \cap \varphi(X)=\left\{\varphi\left(P_{1}\right), \varphi\left(P_{2}\right)\right\}$ if and only if condition (c-ii) is satisfied.

(iii) L is a tangent line at $\varphi\left(P_{2}\right)$ if and only if condition (c-iii) is satisfied.

For the birational embedding $\varphi$ in Theorem 1, we have the following.

THEOREM 2. Let $\varphi$ be as in Theorem 1 , and let $\Lambda$ be the linear system on $X$ corresponding to the morphism $\varphi$. Let $\left(0, \alpha_{P}, \beta_{P}\right)$ denote the $(\Lambda, P)$-order sequence at a point $P \in X$. Then the following hold. 
(1) The multiplicity $m_{\varphi\left(P_{1}\right)}$ of $\varphi(X)$ at $\varphi\left(P_{1}\right)$ is equal to

$$
\left|G_{2}\left(P_{1}\right)\right| \cdot\left|G_{2} \cdot P_{1} \backslash\left(G_{1} \cdot P_{2} \cap G_{2} \cdot P_{1}\right)\right| .
$$

(2) The divisor $\sum_{P \in \varphi^{-1}\left(\varphi\left(P_{1}\right)\right)} \alpha_{P} P$ is equal to

$$
\sum_{Q \in G_{2} \cdot P_{1} \backslash\left(G_{1} \cdot P_{2} \cap G_{2} \cdot P_{1}\right)}\left|G_{2}\left(P_{1}\right)\right| Q .
$$

(3) The multiplicity $m_{\varphi\left(P_{2}\right)}$ of $\varphi(X)$ at $\varphi\left(P_{2}\right)$ is equal to $\left|G_{1}\left(P_{2}\right)\right| \cdot\left|G_{1} \cdot P_{2} \backslash\left(G_{1} \cdot P_{2} \cap G_{2} \cdot P_{1}\right)\right|+\left(\left|G_{1}\left(P_{2}\right)\right|-\left|G_{2}\left(P_{1}\right)\right|\right) \cdot\left|G_{1} \cdot P_{2} \cap G_{2} \cdot P_{1}\right|$.

(4) The divisor $\sum_{P \in \varphi^{-1}\left(\varphi\left(P_{2}\right)\right)} \alpha_{P} P$ is equal to

$$
\sum_{R \in G_{1} \cdot P_{2} \backslash\left(G_{1} \cdot P_{2} \cap G_{2} \cdot P_{1}\right)}\left|G_{1}\left(P_{2}\right)\right| R+\sum_{S \in G_{1} \cdot P_{2} \cap G_{2} \cdot P_{1}}\left(\left|G_{1}\left(P_{2}\right)\right|-\left|G_{2}\left(P_{1}\right)\right|\right) S .
$$

(5) In the case (iii) of Theorem 1, the equality $\beta_{P}=\left|G_{1}\left(P_{2}\right)\right|$ holds at each point $P \in G_{1} \cdot P_{2} \cap G_{2} \cdot P_{1}$.

(6) The divisor $\varphi^{*} L$ is equal to

$$
\sum_{Q \in G_{2} \cdot P_{1} \backslash\left(G_{1} \cdot P_{2} \cap G_{2} \cdot P_{1}\right)}\left|G_{2}\left(P_{1}\right)\right| Q+\sum_{R \in G_{1} \cdot P_{2}}\left|G_{1}\left(P_{2}\right)\right| R .
$$

To explain the usefulness of Theorems 1 and 2, we apply our criterion to rational curves.

THEOREM 3. There exist the following birational embeddings $\varphi: \mathbb{P}^{1} \rightarrow \mathbb{P}^{2}$.

(1) $p=3, \operatorname{deg}\left(\varphi\left(\mathbb{P}^{1}\right)\right)=14$ and there exist two non-smooth Galois points $\varphi\left(P_{1}\right)$ and $\varphi\left(P_{2}\right) \in \varphi\left(\mathbb{P}^{1}\right)$ such that $m_{\varphi\left(P_{1}\right)}=4, m_{\varphi\left(P_{2}\right)}=8, G_{\varphi\left(P_{1}\right)} \cong \mathbf{D}_{5}$, $G_{\varphi\left(P_{2}\right)} \cong \mathrm{AGL}\left(1, \mathbb{F}_{3}\right)$, and $L=\overline{\varphi\left(P_{1}\right) \varphi\left(P_{2}\right)}$ is not a tangent line at $\varphi\left(P_{1}\right)$ and $\varphi\left(P_{2}\right)$. The second order is equal to 2 at each point contained in $\operatorname{supp}\left(\varphi^{*} L\right)$.

(2) $\quad p \neq 2,5, \operatorname{deg}\left(\varphi\left(\mathbb{P}^{1}\right)\right)=16$ and there exist two non-smooth Galois points $\varphi\left(P_{1}\right)$ and $\varphi\left(P_{2}\right) \in \varphi\left(\mathbb{P}^{1}\right)$ such that $m_{\varphi\left(P_{1}\right)}=4, m_{\varphi\left(P_{2}\right)}=11, G_{\varphi\left(P_{1}\right)} \cong \mathbf{A}_{4}$, $G_{\varphi\left(P_{2}\right)} \cong \mathbb{Z} / 5 \mathbb{Z}, L=\overline{\varphi\left(P_{1}\right) \varphi\left(P_{2}\right)}$ is not a tangent line at $\varphi\left(P_{1}\right)$, and $L$ is a tangent line at $\varphi\left(P_{2}\right)$. The second order is equal to 2 (resp. 1) at each point $Q \in G_{\varphi\left(P_{1}\right)} \cdot P_{2} \backslash\left\{P_{2}\right\}$ (resp. $\left.Q \in G_{\varphi\left(P_{2}\right)} \cdot P_{1}\right)$, and the third order is equal to 2 at $P_{2}$.

(3) $\quad p \neq 2,5, \operatorname{deg}\left(\varphi\left(\mathbb{P}^{1}\right)\right)=28$ and there exist two non-smooth Galois points $\varphi\left(P_{1}\right)$ and $\varphi\left(P_{2}\right) \in \varphi\left(\mathbb{P}^{1}\right)$ such that $m_{\varphi\left(P_{1}\right)}=4, m_{\varphi\left(P_{2}\right)}=23, G_{\varphi\left(P_{1}\right)} \cong \mathbf{S}_{4}$, $G_{\varphi\left(P_{2}\right)} \cong \mathbb{Z} / 5 \mathbb{Z}, L=\overline{\varphi\left(P_{1}\right) \varphi\left(P_{2}\right)}$ is not a tangent line at $\varphi\left(P_{1}\right)$, and $L$ is a tangent line at $\varphi\left(P_{2}\right)$. The second order is equal to 4 (resp. 3, 1) 
at each point $Q \in G_{\varphi\left(P_{1}\right)} \cdot P_{2} \backslash\left\{P_{2}\right\} \quad$ (resp. at $P_{2}$, at each point $Q \in$ $\left.G_{\varphi\left(P_{2}\right)} \cdot P_{1} \backslash\left\{P_{2}\right\}\right)$, and the third order is equal to 4 at $P_{2}$.

\section{Preliminaries}

We recall some notation and facts. Let $\varphi: X \rightarrow \mathbb{P}^{2}$ be a morphism, which is birational onto its image. Such a morphism $\varphi$ is called a birational embedding of $X$ to $\mathbb{P}^{2}$. Assume that $\varphi(X)$ is not a line. First, we recall the notion of order sequences (see [10, Chapter 7]). For a line $L \subset \mathbb{P}^{2}$, the intersection divisor of $\varphi(X)$ and $L$ on $X$ is denoted by $\varphi^{*} L$. Note that

$$
\Lambda=\left\{\varphi^{*} L \mid L \text { is a line contained in } \mathbb{P}^{2}\right\}
$$

is the linear system on $X$ corresponding to the morphism $\varphi$. The support of the divisor $\varphi^{*} L$ is denoted by $\operatorname{supp}\left(\varphi^{*} L\right)$. For a point $P \in X$, the order of $\varphi^{*} L$ at $P$ is denoted by $\operatorname{ord}_{P}\left(\varphi^{*} L\right)$. We put

$$
\alpha_{P}=\min \left\{\operatorname{ord}_{P}\left(\varphi^{*} L\right) \mid \varphi^{*} L \in \Lambda, P \in \operatorname{supp}\left(\varphi^{*} L\right)\right\} .
$$

Then there exists a unique line $\tilde{L}$ such that $\beta_{P}=\operatorname{ord}_{P}\left(\varphi^{*} \tilde{L}\right)>\alpha_{P}$. We call the line $\tilde{L}$ the osculating line at $P$, and we call the sequence $\left(0, \alpha_{P}, \beta_{P}\right)$ the $(\Lambda, P)$ order sequence at $P$. A line $\tilde{L}$ passing through $\varphi(P)$ is called a tangent line at $\varphi(P)$ if $\tilde{L}$ is the osculating line at a point contained in $\varphi^{-1}(\varphi(P))$. Note that a line $\tilde{L}$ is a tangent line at $\varphi(P)$ if and only if $m_{\varphi(P)}<I_{\varphi(P)}(\varphi(X), \tilde{L})$, where $I_{\varphi(P)}(\varphi(X), \tilde{L})$ is the intersection multiplicity of $\varphi(X)$ and $\tilde{L}$ at $\varphi(P)$.

Next, we consider the projection $\pi_{\varphi(P)}$, and we put $\hat{\pi}_{\varphi(P)}=\pi_{\varphi(P)} \circ \varphi: X \rightarrow$ $\mathbb{P}^{1}$. We recall some properties of a ramification index of $\hat{\pi}_{\varphi(P)}$. We put $\varphi^{-1}(\varphi(P))=\left\{P_{1}, \ldots, P_{n}\right\}$. Let $\left(0, \alpha_{P_{i}}, \beta_{P_{i}}\right)$ be the $\left(\Lambda, P_{i}\right)$-order sequence for $i=1, \ldots, n$. The ramification index of $\hat{\pi}_{\varphi(P)}$ at a point $Q \in X$ is denoted by $e_{Q}\left(\hat{\pi}_{\varphi(P)}\right)$. Then the following fact is well-known.

FACT 4. Let $Q \in X \backslash\left\{P_{1}, \ldots, P_{n}\right\}$.

(1) The equality $e_{Q}\left(\hat{\pi}_{\varphi(P)}\right)=\operatorname{ord}_{Q}\left(\varphi^{*} \overline{\varphi(P) \varphi(Q)}\right)$ holds.

(2) The equality $e_{P_{i}}\left(\hat{\pi}_{\varphi(P)}\right)=\beta_{P_{i}}-\alpha_{P_{i}}$ holds for $i=1, \ldots, n$.

Finally, we recall some properties of a Galois covering for the proof of our main theorems (see [14, III. 7.1, 7.2 and 8.2]).

FACT 5. Let $\theta: X \rightarrow Y$ be a surjective morphism of smooth projective curves, and assume that the field extension $k(X) / \theta^{*} k(Y)$ is a Galois extension with the Galois group $G$. Then the following hold.

(1) If $P, Q \in X$ and $\theta(P)=\theta(Q)$, then there exists an element $\sigma \in G$ such that $\sigma(P)=Q$. 
(2) If $P, Q \in X$ and $\theta(P)=\theta(Q)$, then $e_{P}(\theta)=e_{Q}(\theta)$.

(3) For each point $P \in X$, the order $|G(P)|$ is equal to $e_{P}(\theta)$.

\section{Proofs of Theorems 1 and 2}

The same notation is used as in the previous section. The following lemma shows that Theorem 1 describes all cases with two inner Galois points.

Lemma 6. Let $P_{1}, P_{2} \in X$, and assume that $\varphi\left(P_{1}\right)$ and $\varphi\left(P_{2}\right)$ are different inner Galois points. We put $L=\overline{\varphi\left(P_{1}\right) \varphi\left(P_{2}\right)}$. Then either $m_{\varphi\left(P_{1}\right)}=$ $I_{\varphi\left(P_{1}\right)}(\varphi(X), L)$ or $m_{\varphi\left(P_{2}\right)}=I_{\varphi\left(P_{2}\right)}(\varphi(X), L)$ holds.

Proof. We put

$$
\begin{aligned}
& \varphi^{-1}\left(\varphi\left(P_{1}\right)\right)=\left\{P_{11}=P_{1}, P_{12}, \ldots, P_{1 n_{1}}\right\}, \\
& \varphi^{-1}\left(\varphi\left(P_{2}\right)\right)=\left\{P_{21}=P_{2}, P_{22}, \ldots, P_{2 n_{2}}\right\} .
\end{aligned}
$$

Let $\left(0, \alpha_{P_{i j}}, \beta_{P_{i j}}\right)$ be the $\left(\Lambda, P_{i j}\right)$-order sequence for $i, j$. Assume by contradiction that

$$
m_{\varphi\left(P_{1}\right)}<I_{\varphi\left(P_{1}\right)}(\varphi(X), L) \quad \text { and } \quad m_{\varphi\left(P_{2}\right)}<I_{\varphi\left(P_{2}\right)}(\varphi(X), L)
$$

hold. By Fact 5, the ramification index of $\hat{\pi}_{\varphi\left(P_{1}\right)}$ (resp. $\left.\hat{\pi}_{\varphi\left(P_{2}\right)}\right)$ at each point contained in $\varphi^{-1}\left(\varphi\left(P_{2}\right)\right)$ (resp. $\left.\varphi^{-1}\left(\varphi\left(P_{1}\right)\right)\right)$ coincides with $\left|G_{\varphi\left(P_{1}\right)}\left(P_{2}\right)\right|$ (resp. $\left.\left|G_{\varphi\left(P_{2}\right)}\left(P_{1}\right)\right|\right)$. By Fact $4(1)$ and Fact 5, $\left|G_{\varphi\left(P_{1}\right)}\left(P_{2}\right)\right|$ (resp. $\left.\left|G_{\varphi\left(P_{2}\right)}\left(P_{1}\right)\right|\right)$ coincides with $\operatorname{ord}_{P_{2 j}}\left(\varphi^{*} L\right)$ for each $j\left(\right.$ resp. $\operatorname{ord}_{P_{1 i}}\left(\varphi^{*} L\right)$ for each $\left.i\right)$. Since $L$ is a tangent line at $\varphi\left(P_{1}\right)$ (resp. $\left.\varphi\left(P_{2}\right)\right)$, there exists $i_{0}$ (resp. $\left.j_{0}\right)$ such that

$$
\beta_{P_{1 i_{0}}}=\operatorname{ord}_{P_{1_{0}}}\left(\varphi^{*} L\right) \quad\left(\text { resp. } \beta_{P_{2 j_{0}}}=\operatorname{ord}_{P_{2 j_{0}}}\left(\varphi^{*} L\right)\right) .
$$

By Fact 4 (2) and Fact 5,

$$
\left|G_{\varphi\left(P_{1}\right)}\left(P_{2}\right)\right|=\beta_{P_{1 i_{0}}}-\alpha_{P_{1 i_{0}}} \quad\left(\text { resp. }\left|G_{\varphi\left(P_{2}\right)}\left(P_{1}\right)\right|=\beta_{P_{2 j_{0}}}-\alpha_{P_{2 j_{0}}}\right)
$$

holds. Therefore, we have a contradiction as follows:

$$
\begin{aligned}
\left|G_{\varphi\left(P_{2}\right)}\left(P_{1}\right)\right| & <\left|G_{\varphi\left(P_{2}\right)}\left(P_{1}\right)\right|+\alpha_{P_{2 j_{0}}}=\beta_{P_{2 j_{0}}}=\operatorname{ord}_{P_{2 j_{0}}}\left(\varphi^{*} L\right)=\left|G_{\varphi\left(P_{1}\right)}\left(P_{2}\right)\right| \\
& <\left|G_{\varphi\left(P_{1}\right)}\left(P_{2}\right)\right|+\alpha_{P_{1_{0}}}=\beta_{P_{1_{i_{0}}}}=\operatorname{ord}_{P_{1_{0}}}\left(\varphi^{*} L\right)=\left|G_{\varphi\left(P_{2}\right)}\left(P_{1}\right)\right| .
\end{aligned}
$$

Proof (Proof of Theorem 1). We consider the 'if' part. Assume that conditions (a), (b), and (c) in Theorem 1 are satisfied. Let $f, g \in k(X)$ be the generators of $k(X)^{G_{1}}$ and $k(X)^{G_{2}}$ such that

$$
(f)_{\infty}=\sum_{\sigma \in G_{1}} \sigma\left(P_{2}\right), \quad(g)_{\infty}=\sum_{\tau \in G_{2}} \tau\left(P_{1}\right),
$$


which exist by condition $(\mathrm{a})$, where $(f)_{\infty}\left(\right.$ resp. $\left.(g)_{\infty}\right)$ is the pole divisor of $f$ (resp. $g)$. We consider the morphism $\varphi=(f: g: 1): X \rightarrow \mathbb{P}^{2}$. First, we show that the equality $\varphi\left(P_{1}\right)=(0: 1: 0)$ holds. We put $n_{g}=\operatorname{ord}_{P_{1}}\left((g)_{\infty}\right)$. Note that $n_{g}$ is equal to $\left|G_{2}\left(P_{1}\right)\right|$. Let $t_{P_{1}}$ be a local parameter at $P_{1}$. Since $P_{1} \notin G_{1} \cdot P_{2}=\operatorname{supp}\left((f)_{\infty}\right)$ by condition (c),

$$
\operatorname{ord}_{P_{1}}\left(t_{P_{1}}^{n_{g}} f\right)=n_{g}+\operatorname{ord}_{P_{1}}(f) \geq n_{g}>0
$$

hold. Therefore, we have the equality $\varphi\left(P_{1}\right)=(0: 1: 0)$. We also show that the equality $\varphi\left(P_{2}\right)=(1: 0: 0)$ holds. We put $n_{f}=\operatorname{ord}_{P_{2}}\left((f)_{\infty}\right)$. Note that $n_{f}$ is equal to $\left|G_{1}\left(P_{2}\right)\right|$. Let $t_{P_{2}}$ be a local parameter at $P_{2}$. If $P_{2} \notin G_{2} \cdot P_{1}=$ $\operatorname{supp}\left((g)_{\infty}\right)$, we have

$$
\operatorname{ord}_{P_{2}}\left(t_{P_{2}}^{n_{f}} g\right)=n_{f}+\operatorname{ord}_{P_{2}}(g) \geq n_{f}>0 .
$$

If $P_{2} \in G_{2} \cdot P_{1}$, then condition (c-iii) is satisfied, and we have

$$
\operatorname{ord}_{P_{2}}\left(t_{P_{2}}^{n_{f}} g\right)=n_{f}+\operatorname{ord}_{P_{2}}(g)=\left|G_{1}\left(P_{2}\right)\right|-\left|G_{2}\left(P_{1}\right)\right|>0 .
$$

Therefore, the equality $\varphi\left(P_{2}\right)=(1: 0: 0)$ holds. By a method similar to the proof of [5, Proposition 1], by condition (b), we can show that the morphism $\varphi$ is birational onto its image. The morphism $(f: 1)$ (resp. $(g: 1))$ coincides with the projection from the point

$$
\varphi\left(P_{1}\right)=(0: 1: 0) \quad\left(\text { resp. } \varphi\left(P_{2}\right)=(1: 0: 0)\right) .
$$

Therefore $\varphi\left(P_{1}\right)$ and $\varphi\left(P_{2}\right)$ are different inner Galois points, and $G_{\varphi\left(P_{i}\right)}=G_{i}$ for $i=1,2$. We show that $L=\overline{\varphi\left(P_{1}\right) \varphi\left(P_{2}\right)}$ is not a tangent line at $\varphi\left(P_{1}\right)$. Assume by contradiction that $L$ is a tangent line at $\varphi\left(P_{1}\right)$. Then there exists a point $Q \in \varphi^{-1}\left(\varphi\left(P_{1}\right)\right)$ such that $Q \in G_{1} \cdot P_{2}$. Let $\Lambda$ be the linear system on $X$ corresponding to the morphism $\varphi$, and let $\left(0, \alpha_{Q}, \beta_{Q}\right)$ be the $(\Lambda, Q)$-order sequence at $Q$. Since $L$ is the osculating line at $Q$, we have

$$
\left|G_{2}\left(P_{1}\right)\right|=\operatorname{ord}_{Q}\left(\varphi^{*} L\right)=\beta_{Q}
$$

by Fact 4 (1) and Fact 5 . On the other hand, by Fact 4 (2) and Fact 5 , the equality

$$
\left|G_{1}\left(P_{2}\right)\right|=\beta_{Q}-\alpha_{Q}
$$

holds. Therefore, we have $G_{1} \cdot P_{2} \cap G_{2} \cdot P_{1} \neq \varnothing$ and $\left|G_{1}\left(P_{2}\right)\right|<\left|G_{2}\left(P_{1}\right)\right|$. This is a contradiction to condition (c). Therefore, $L$ is not a tangent line at $\varphi\left(P_{1}\right)$.

We consider the 'only if' part. Assume that there exists a birational embedding $\varphi: X \rightarrow \mathbb{P}^{2}$ such that $\varphi\left(P_{1}\right)$ and $\varphi\left(P_{2}\right)$ are different inner Galois 
points, $G_{\varphi\left(P_{i}\right)}=G_{i}$ for $i=1,2$, and $L=\overline{\varphi\left(P_{1}\right) \varphi\left(P_{2}\right)}$ is not a tangent line at $\varphi\left(P_{1}\right)$. Since

$$
k(X)^{G_{i}}=\left(\hat{\pi}_{\varphi\left(P_{i}\right)}\right)^{*}\left(k\left(\mathbb{P}^{1}\right)\right) \cong k\left(\mathbb{P}^{1}\right)
$$

for $i=1,2$, condition (a) is satisfied. By a method similar to the proof of $[5$, Theorem 1], condition (b) is satisfied. Since $L$ is not a tangent line at $\varphi\left(P_{1}\right)$, we have $P_{1} \notin G_{1} \cdot P_{2}$. We show condition (c) by dividing into the following three cases (I), (II), and (III).

(I) Assume that $L$ is not a tangent line at $\varphi\left(P_{2}\right)$ and

$$
L \cap \varphi(X) \supsetneq\left\{\varphi\left(P_{1}\right), \varphi\left(P_{2}\right)\right\} .
$$

We show that condition (c-i) is satisfied. Since $L$ is not a tangent line at $\varphi\left(P_{2}\right)$ and $(L \cap \varphi(X)) \backslash\left\{\varphi\left(P_{1}\right), \varphi\left(P_{2}\right)\right\} \neq \varnothing$, we have $P_{2} \notin G_{2} \cdot P_{1}$ and $G_{1} \cdot P_{2} \cap G_{2} \cdot P_{1}$ $\neq \varnothing$. We take a point

$$
Q \in \varphi^{-1}\left((L \cap \varphi(X)) \backslash\left\{\varphi\left(P_{1}\right), \varphi\left(P_{2}\right)\right\}\right) .
$$

By Fact 4 (1) and Fact 5, we have the equalities

$$
\left|G_{1}\left(P_{2}\right)\right|=\operatorname{ord}_{Q}\left(\varphi^{*} L\right)=\left|G_{2}\left(P_{1}\right)\right| .
$$

Therefore, condition (c-i) is satisfied.

(II) Assume that $L$ is not a tangent line at $\varphi\left(P_{2}\right)$ and

$$
L \cap \varphi(X)=\left\{\varphi\left(P_{1}\right), \varphi\left(P_{2}\right)\right\} .
$$

Then $G_{1} \cdot P_{2}=\varphi^{-1}\left(\varphi\left(P_{2}\right)\right)$ and $G_{2} \cdot P_{1}=\varphi^{-1}\left(\varphi\left(P_{1}\right)\right)$ hold, and we have

$$
G_{1} \cdot P_{2} \cap G_{2} \cdot P_{1}=\varnothing .
$$

Therefore, condition (c-ii) is satisfied.

(III) Assume that $L$ is a tangent line at $\varphi\left(P_{2}\right)$. We show that condition (c-iii) is satisfied. Since $L$ is a tangent line at $\varphi\left(P_{2}\right)$, there exists a point $Q \in \varphi^{-1}\left(\varphi\left(P_{2}\right)\right)$ such that $Q \in G_{2} \cdot P_{1}$. Since $G_{1} \cdot P_{2} \supset \varphi^{-1}\left(\varphi\left(P_{2}\right)\right)$, we have $G_{1} \cdot P_{2} \cap G_{2} \cdot P_{1} \neq \varnothing$. Let $\Lambda$ be the linear system on $X$ corresponding to the morphism $\varphi$, and let $\left(0, \alpha_{Q}, \beta_{Q}\right)$ be the $(\Lambda, Q)$-order sequence at $Q$. Since $L$ is the osculating line at $Q$, we have

$$
\left|G_{1}\left(P_{2}\right)\right|=\operatorname{ord}_{Q}\left(\varphi^{*} L\right)=\beta_{Q}
$$

by Fact 4 (1) and Fact 5 . On the other hand, by Fact 4 (2) and Fact 5 , the equality

$$
\left|G_{2}\left(P_{1}\right)\right|=\beta_{Q}-\alpha_{Q}
$$


holds, and we have $\left|G_{1}\left(P_{2}\right)\right|>\left|G_{2}\left(P_{1}\right)\right|$. Therefore, condition (c-iii) is satisfied.

Finally, we show (i), (ii), and (iii) in Theorem 1. Let $\varphi$ be as in Theorem 1. Then condition (c-i), (c-ii), or (c-iii) is satisfied. Since these conditions are mutually exclusive, it is enough to show 'only if' part of (i), (ii), and (iii) in Theorem 1. This task has been already done above.

Proof (Proof of Theorem 2). Let $\varphi$ be as in Theorem 1, and let $\Lambda$ be the linear system on $X$ corresponding to the morphism $\varphi$. We put

$$
\begin{aligned}
& \varphi^{-1}\left(\varphi\left(P_{1}\right)\right)=\left\{P_{11}=P_{1}, P_{12}, \ldots, P_{1 n_{1}}\right\}, \\
& \varphi^{-1}\left(\varphi\left(P_{2}\right)\right)=\left\{P_{21}=P_{2}, P_{22}, \ldots, P_{2 n_{2}}\right\} .
\end{aligned}
$$

Let $\left(0, \alpha_{P_{i j}}, \beta_{P_{i j}}\right)$ be the $\left(\Lambda, P_{i j}\right)$-order sequence for $i, j$.

First, we show Theorem 2 (1) and (2). Since the linear system corresponding to the morphism $\hat{\pi}_{\varphi\left(P_{1}\right)}$ is

$$
\left\{E-\sum_{i=1}^{n_{1}} \alpha_{P_{1 i}} P_{1 i} \mid E \in \Lambda, E \geq \sum_{i=1}^{n_{1}} \alpha_{P_{1 i}} P_{1 i}\right\}
$$

and $\hat{\pi}_{\varphi\left(P_{1}\right)}$ is a Galois covering, the following equalities of divisors hold:

$$
\varphi^{*} L-\sum_{i=1}^{n_{1}} \alpha_{P_{1 i}} P_{1 i}=\left(\hat{\pi}_{\varphi\left(P_{1}\right)}\right)^{*}([L])=\sum_{\sigma \in G_{1}} \sigma\left(P_{2}\right),
$$

where $[L]$ represents the divisor of the point $[L] \in \mathbb{P}^{1}$ corresponding to the line $L$. By Fact $4(1)$ and Fact 5 , the equality $\left|G_{2}\left(P_{1}\right)\right|=\operatorname{ord}_{P_{1 i}}\left(\varphi^{*} L\right)$ holds for all $i$. Since $L$ is not a tangent line at $\varphi\left(P_{1}\right)$, the equality $\alpha_{P_{1 i}}=\left|G_{2}\left(P_{1}\right)\right|$ holds for all $i$. It is not difficult to check that

$$
\left(\varphi^{-1}\left(\varphi\left(P_{1}\right)\right)\right) \cup\left(G_{1} \cdot P_{2}\right)=\operatorname{supp}\left(\varphi^{*} L\right)=\left(G_{2} \cdot P_{1}\right) \cup\left(G_{1} \cdot P_{2}\right)
$$

hold. Since the intersection of the two sets $\varphi^{-1}\left(\varphi\left(P_{1}\right)\right)$ and $G_{1} \cdot P_{2}$ is the empty set, we have

$$
\begin{aligned}
\varphi^{-1}\left(\varphi\left(P_{1}\right)\right) & =\left(\left(\varphi^{-1}\left(\varphi\left(P_{1}\right)\right)\right) \cup\left(G_{1} \cdot P_{2}\right)\right) \backslash\left(G_{1} \cdot P_{2}\right) \\
& =G_{2} \cdot P_{1} \backslash\left(G_{1} \cdot P_{2} \cap G_{2} \cdot P_{1}\right) .
\end{aligned}
$$

Therefore, the equality

$$
\sum_{i=1}^{n_{1}} \alpha_{P_{1 i}} P_{1 i}=\sum_{Q \in G_{2} \cdot P_{1} \backslash\left(G_{1} \cdot P_{2} \cap G_{2} \cdot P_{1}\right)}\left|G_{2}\left(P_{1}\right)\right| Q
$$


of divisors holds, and we have Theorem 2 (2). Since

$$
m_{\varphi\left(P_{1}\right)}=\sum_{i=1}^{n_{1}} \alpha_{P_{1 i}}
$$

we have Theorem 2 (1).

Next, we show Theorem 2 (6). By the above, the equality

$$
\varphi^{*} L=\sum_{Q \in G_{2} \cdot P_{1} \backslash\left(G_{1} \cdot P_{2} \cap G_{2} \cdot P_{1}\right)}\left|G_{2}\left(P_{1}\right)\right| Q+\sum_{\sigma \in G_{1}} \sigma\left(P_{2}\right)
$$

holds. Since

$$
\sum_{\sigma \in G_{1}} \sigma\left(P_{2}\right)=\sum_{R \in G_{1} \cdot P_{2}}\left|G_{1}\left(P_{2}\right)\right| R
$$

we have Theorem 2 (6).

Finally, we show Theorem 2 (3), (4), and (5). Since

$$
\sum_{\tau \in G_{2}} \tau\left(P_{1}\right)=\sum_{S \in G_{1} \cdot P_{2} \cap G_{2} \cdot P_{1}}\left|G_{2}\left(P_{1}\right)\right| S+\sum_{Q \in G_{2} \cdot P_{1} \backslash\left(G_{1} \cdot P_{2} \cap G_{2} \cdot P_{1}\right)}\left|G_{2}\left(P_{1}\right)\right| Q
$$

the following equalities of divisors hold:

$$
\begin{aligned}
& \sum_{R \in G_{1} \cdot P_{2} \backslash\left(G_{1} \cdot P_{2} \cap G_{2} \cdot P_{1}\right)}\left|G_{1}\left(P_{2}\right)\right| R \\
& +\sum_{S \in G_{1} \cdot P_{2} \cap G_{2} \cdot P_{1}}\left(\left|G_{1}\left(P_{2}\right)\right|-\left|G_{2}\left(P_{1}\right)\right|\right) S+\sum_{\tau \in G_{2}} \tau\left(P_{1}\right) \\
= & \left.\sum_{R \in G_{1} \cdot P_{2} \backslash\left(G_{1} \cdot P_{2} \cap G_{2} \cdot P_{1}\right)}\left|G_{1}\left(P_{2}\right)\right| R+\sum_{S \in G_{1} \cdot P_{2} \cap G_{2} \cdot P_{1}}\left|G_{1}\left(P_{2}\right)\right| S\right) \\
& +\sum_{Q \in G_{2} \cdot P_{1} \backslash\left(G_{1} \cdot P_{2} \cap G_{2} \cdot P_{1}\right)}\left|G_{2}\left(P_{1}\right)\right| Q \\
= & \sum_{Q \in G_{2} \cdot P_{1} \backslash\left(G_{1} \cdot P_{2} \cap G_{2} \cdot P_{1}\right)}\left|G_{2}\left(P_{1}\right)\right| Q+\sum_{R \in G_{1} \cdot P_{2}}\left|G_{1}\left(P_{2}\right)\right| R \\
= & \varphi^{*} L, \sum
\end{aligned}
$$

where the last equality comes from Theorem 2 (6). Therefore, the equality

$$
\begin{aligned}
\varphi^{*} L-\sum_{\tau \in G_{2}} \tau\left(P_{1}\right)= & \sum_{R \in G_{1} \cdot P_{2} \backslash\left(G_{1} \cdot P_{2} \cap G_{2} \cdot P_{1}\right)}\left|G_{1}\left(P_{2}\right)\right| R \\
& +\sum_{S \in G_{1} \cdot P_{2} \cap G_{2} \cdot P_{1}}\left(\left|G_{1}\left(P_{2}\right)\right|-\left|G_{2}\left(P_{1}\right)\right|\right) S
\end{aligned}
$$


of divisors holds. On the other hand, since the linear system corresponding to the morphism $\hat{\pi}_{\varphi\left(P_{2}\right)}$ is

$$
\left\{E-\sum_{j=1}^{n_{2}} \alpha_{P_{2 j}} P_{2 j} \mid E \in \Lambda, E \geq \sum_{j=1}^{n_{2}} \alpha_{P_{2 j}} P_{2 j}\right\}
$$

and $\hat{\pi}_{\varphi\left(P_{2}\right)}$ is a Galois covering, the following equalities of divisors hold:

$$
\varphi^{*} L-\sum_{j=1}^{n_{2}} \alpha_{P_{2 j}} P_{2 j}=\left(\hat{\pi}_{\varphi\left(P_{2}\right)}\right)^{*}([L])=\sum_{\tau \in G_{2}} \tau\left(P_{1}\right) .
$$

Therefore, the equalities

$$
\begin{aligned}
\sum_{j=1}^{n_{2}} \alpha_{P_{2 j}} P_{2 j}= & \varphi^{*} L-\sum_{\tau \in G_{2}} \tau\left(P_{1}\right) \\
= & \sum_{R \in G_{1} \cdot P_{2} \backslash\left(G_{1} \cdot P_{2} \cap G_{2} \cdot P_{1}\right)}\left|G_{1}\left(P_{2}\right)\right| R \\
& +\sum_{S \in G_{1} \cdot P_{2} \cap G_{2} \cdot P_{1}}\left(\left|G_{1}\left(P_{2}\right)\right|-\left|G_{2}\left(P_{1}\right)\right|\right) S
\end{aligned}
$$

of divisors hold, and we have Theorem 2 (4). Since

$$
m_{\varphi\left(P_{2}\right)}=\sum_{j=1}^{n_{2}} \alpha_{P_{2 j}}
$$

we have Theorem 2 (3). Assume that the condition (c-iii) in Theorem 1 is satisfied. Then

$$
0<\left|G_{1}\left(P_{2}\right)\right|-\left|G_{2}\left(P_{1}\right)\right|<\left|G_{1}\left(P_{2}\right)\right|
$$

hold. By Theorem $2(6)$, the equality $\left|G_{1}\left(P_{2}\right)\right|=\operatorname{ord}_{P}\left(\varphi^{*} L\right)$ holds at each point $P \in G_{1} \cdot P_{2}$. By Theorem 2 (4), the second $(\Lambda, P)$-order coincides with $\left|G_{1}\left(P_{2}\right)\right|-\left|G_{2}\left(P_{1}\right)\right|$ at each point $P \in G_{1} \cdot P_{2} \cap G_{2} \cdot P_{1}$. Therefore, the third $(\Lambda, P)$-order coincides with $\left|G_{1}\left(P_{2}\right)\right|$ at each point $P \in G_{1} \cdot P_{2} \cap G_{2} \cdot P_{1}$, and Theorem 2 (5) holds.

REMARK 7. In [7], Fukasawa presented a criterion for the existence of a birational embedding with a pair of Galois points consisting of a smooth Galois point and an outer Galois point. By a method similar to the proof of Theorems 1 and 2, we can extend the criterion to non-smooth and outer Galois points. The necessary and sufficient conditions for the existence of a birational embedding with inner and outer Galois points are that $X / G_{i} \cong \mathbb{P}^{1}$ for $i=1,2$, 
$G_{1} \cap G_{2}=\{1\}$, and there exist $\eta \in G_{2}$ and $P \in X$ such that

$$
\left|G_{2}(P)\right| \sum_{Q \in\left(G_{2} \cdot P\right)-\left(G_{1} \cdot \eta(P)\right)} Q+\left(\left|G_{2}(P)\right|-\left|G_{1}(\eta(P))\right|\right) \sum_{R \in G_{1} \cdot \eta(P)} R \geq P .
$$

\section{Proof of Theorem 3}

We apply Theorems 1 and 2 to rational curves. In this case, condition (a) in Theorem 1 is always satisfied, by Lüroth's theorem. We identify $\operatorname{Aut}\left(\mathbb{P}^{1}\right)$ with the projective linear group $\operatorname{PGL}(2, k)$. We put $Q_{\infty}=(1: 0)$ and $Q_{a}=$ $(a: 1) \in \mathbb{P}^{1}$ for any $a \in k$.

Proof (Proof of Theorem 3). Let $p \neq 2,5$, let $i \in k$ be a root of the polynomial $T^{2}+1 \in k[T]$, and let $\xi$ be a primitive fifth root of unity.

(1) Let $p=3$, and let $P_{1}=Q_{0}$ and $P_{2}=Q_{\xi}$. We consider two sets:

$$
G_{1}=\left\langle\left[\begin{array}{ll}
\xi & 0 \\
0 & 1
\end{array}\right]\right\rangle\left\langle\left[\begin{array}{ll}
0 & 1 \\
1 & 0
\end{array}\right]\right\rangle \quad \text { and } \quad G_{2}=\left\langle\left[\begin{array}{ll}
1 & 1 \\
0 & 1
\end{array}\right]\right\rangle\left\langle\left[\begin{array}{cc}
1 & 0 \\
0 & -1
\end{array}\right]\right\rangle \text {. }
$$

It is known that

$$
G_{1}=\left\langle\left[\begin{array}{ll}
\xi & 0 \\
0 & 1
\end{array}\right]\right\rangle \rtimes\left\langle\left[\begin{array}{ll}
0 & 1 \\
1 & 0
\end{array}\right]\right\rangle \cong \mathbf{D}_{5}
$$

where $\mathbf{D}_{5}$ is the dihedral group of degree 5 (see [2, Theorem C]). It is not difficult to check that

$$
G_{2}=\left\langle\left[\begin{array}{ll}
1 & 1 \\
0 & 1
\end{array}\right]\right\rangle \rtimes\left\langle\left[\begin{array}{cc}
1 & 0 \\
0 & -1
\end{array}\right]\right\rangle \cong \operatorname{AGL}\left(1, \mathbb{F}_{3}\right),
$$

where $\operatorname{AGL}\left(1, \mathbb{F}_{3}\right)$ is the general affine group of degree 1 over $\mathbb{F}_{3}$. By direct computations, we have the equalities

$$
\begin{gathered}
G_{1} \cap G_{2}=\{1\}, \\
G_{1} \cdot P_{2}=\left\{Q_{1}, Q_{\xi}, Q_{\xi^{2}}, Q_{\xi^{3}}, Q_{\xi^{4}}\right\}, \\
G_{2} \cdot P_{1}=\left\{Q_{-1}, Q_{0}, Q_{1}\right\}, \\
G_{1} \cdot P_{2} \cap G_{2} \cdot P_{1}=\left\{Q_{1}\right\}, \\
G_{1}\left(P_{2}\right)=\left\{\left[\begin{array}{ll}
1 & 0 \\
0 & 1
\end{array}\right],\left[\begin{array}{cc}
0 & \xi^{2} \\
1 & 0
\end{array}\right]\right\}, \quad \text { and } \\
G_{2}\left(P_{1}\right)=\left\{\left[\begin{array}{ll}
1 & 0 \\
0 & 1
\end{array}\right],\left[\begin{array}{cc}
-1 & 0 \\
0 & 1
\end{array}\right]\right\} .
\end{gathered}
$$


Therefore, conditions (b) and (c-i) in Theorem 1 are satisfied, and there exists a birational embedding $\varphi: \mathbb{P}^{1} \rightarrow \mathbb{P}^{2}$ such that $\varphi\left(P_{1}\right)$ and $\varphi\left(P_{2}\right)$ are different inner Galois points, $G_{\varphi\left(P_{1}\right)} \cong \mathbf{D}_{5}, G_{\varphi\left(P_{2}\right)} \cong \operatorname{AGL}\left(1, \mathbb{F}_{3}\right)$, and $L=\overline{\varphi\left(P_{1}\right) \varphi\left(P_{2}\right)}$ is not a tangent line at $\varphi\left(P_{1}\right), \varphi\left(P_{2}\right)$. By Theorem 2 (1), (3) and (6), $m_{\varphi\left(P_{1}\right)}=4$, $m_{\varphi\left(P_{2}\right)}=8$ and $\operatorname{deg}\left(\varphi\left(\mathbb{P}^{1}\right)\right)=14$. By Theorem $2(6)$, the second order is equal to 2 at each point contained in $\operatorname{supp}\left(\varphi^{*} L\right)$.

(2) Let $P_{1}=Q_{\xi}$ and $P_{2}=Q_{1}$. We consider

$$
G_{1}=\left\langle\left[\begin{array}{cc}
1 & 0 \\
0 & -1
\end{array}\right],\left[\begin{array}{cc}
0 & 1 \\
1 & 0
\end{array}\right]\right\rangle\left\langle\left[\begin{array}{cc}
1 & i \\
1 & -i
\end{array}\right]\right\rangle \quad \text { and } \quad G_{2}=\left\langle\left[\begin{array}{ll}
\xi & 0 \\
0 & 1
\end{array}\right]\right\rangle \text {. }
$$

Obviously, $G_{2} \cong \mathbb{Z} / 5 \mathbb{Z}$, and the following fact is known.

$$
G_{1}=\left\langle\left[\begin{array}{cc}
1 & 0 \\
0 & -1
\end{array}\right],\left[\begin{array}{ll}
0 & 1 \\
1 & 0
\end{array}\right]\right\rangle \rtimes\left\langle\left[\begin{array}{cc}
1 & i \\
1 & -i
\end{array}\right]\right\rangle \cong \mathbf{A}_{4},
$$

where $\mathbf{A}_{4}$ is the alternating group of degree 4 (see [2, Theorem C]). Since 5 and 12 are coprime, condition (b) in Theorem 1 is satisfied. By direct computations, we have the following equalities:

$$
\begin{gathered}
G_{1} \cdot P_{2}=\left\{Q_{-i}, Q_{-1}, Q_{0}, Q_{1}, Q_{i}, Q_{\infty}\right\}, \\
G_{2} \cdot P_{1}=\left\{Q_{1}, Q_{\xi}, Q_{\xi^{2}}, Q_{\xi^{3}}, Q_{\xi^{4}}\right\}, \\
G_{1} \cdot P_{2} \cap G_{2} \cdot P_{1}=\left\{Q_{1}=P_{2}\right\}, \\
G_{1}\left(P_{2}\right)=\left\{\left[\begin{array}{ll}
1 & 0 \\
0 & 1
\end{array}\right],\left[\begin{array}{ll}
0 & 1 \\
1 & 0
\end{array}\right]\right\}, \quad \text { and } \\
G_{2}\left(P_{1}\right)=\left\{\left[\begin{array}{ll}
1 & 0 \\
0 & 1
\end{array}\right]\right\} .
\end{gathered}
$$

Therefore, condition (c-iii) in Theorem 1 is satisfied, and there exists a birational embedding $\varphi: \mathbb{P}^{1} \rightarrow \mathbb{P}^{2}$ such that $\varphi\left(P_{1}\right)$ and $\varphi\left(P_{2}\right)$ are different inner Galois points, $G_{\varphi\left(P_{1}\right)} \cong \mathbf{A}_{4}, G_{\varphi\left(P_{2}\right)} \cong \mathbb{Z} / 5 \mathbb{Z}, L=\overline{\varphi\left(P_{1}\right) \varphi\left(P_{2}\right)}$ is not a tangent line at $\varphi\left(P_{1}\right)$, and $L$ is a tangent line at $\varphi\left(P_{2}\right)$. By Theorem 2 (1), (3) and (6), $m_{\varphi\left(P_{1}\right)}=4, m_{\varphi\left(P_{2}\right)}=11$ and $\operatorname{deg}\left(\varphi\left(\mathbb{P}^{1}\right)\right)=16$. By Theorem 2 (2), (4), and (5), the second order is equal to 2 (resp. 1) at each point $Q \in G_{1} \cdot P_{2} \backslash\left\{P_{2}\right\}$ (resp. $\left.Q \in G_{2} \cdot P_{1}\right)$, and the third order is equal to 2 at $P_{2}$.

(3) Let $P_{1}=Q_{\xi}$ and $P_{2}=Q_{1}$. We consider two groups:

$$
\begin{gathered}
G_{1}=\left\langle\left\langle\left[\begin{array}{cc}
1 & 0 \\
0 & -1
\end{array}\right],\left[\begin{array}{ll}
0 & 1 \\
1 & 0
\end{array}\right]\right\rangle \rtimes\left\langle\left[\begin{array}{cc}
1 & i \\
1 & -i
\end{array}\right]\right\rangle,\left\langle\left[\begin{array}{ll}
i & 0 \\
0 & 1
\end{array}\right]\right\rangle\right\rangle, \\
G_{2}=\left\langle\left[\begin{array}{ll}
\xi & 0 \\
0 & 1
\end{array}\right]\right\rangle .
\end{gathered}
$$


Obviously, $G_{2} \cong \mathbb{Z} / 5 \mathbb{Z}$, and the following fact is known:

$$
\left\langle\left[\begin{array}{cc}
1 & 0 \\
0 & -1
\end{array}\right],\left[\begin{array}{ll}
0 & 1 \\
1 & 0
\end{array}\right]\right\rangle \rtimes\left\langle\left[\begin{array}{cc}
1 & i \\
1 & -i
\end{array}\right]\right\rangle \triangleleft G_{1} \cong \mathbf{S}_{4},
$$

where $\mathbf{S}_{4}$ is the symmetric group of degree 4 (see [2, Theorem C]). Since 5 and 24 are coprime, condition (b) in Theorem 1 is satisfied. By direct computations, we have the following equalities:

$$
\begin{gathered}
G_{1} \cdot P_{2}=\left\{Q_{-i}, Q_{-1}, Q_{0}, Q_{1}, Q_{i}, Q_{\infty}\right\}, \\
G_{2} \cdot P_{1}=\left\{Q_{1}, Q_{\xi}, Q_{\xi^{2}}, Q_{\xi^{3}}, Q_{\xi^{4}}\right\}, \\
G_{1} \cdot P_{2} \cap G_{2} \cdot P_{1}=\left\{Q_{1}=P_{2}\right\}, \\
G_{1}\left(P_{2}\right)=\left\{\left[\begin{array}{ll}
1 & 0 \\
0 & 1
\end{array}\right],\left[\begin{array}{ll}
0 & 1 \\
1 & 0
\end{array}\right],\left[\begin{array}{ll}
i & 1 \\
1 & i
\end{array}\right],\left[\begin{array}{ll}
1 & i \\
i & 1
\end{array}\right]\right\}, \quad \text { and } \\
G_{2}\left(P_{1}\right)=\left\{\left[\begin{array}{ll}
1 & 0 \\
0 & 1
\end{array}\right]\right\} .
\end{gathered}
$$

Therefore, condition (c-iii) in Theorem 1 is satisfied, and there exists a birational embedding $\varphi: \mathbb{P}^{1} \rightarrow \mathbb{P}^{2}$ such that $\varphi\left(P_{1}\right)$ and $\varphi\left(P_{2}\right)$ are different inner Galois points, $G_{\varphi\left(P_{1}\right)} \cong \mathbf{S}_{4}, G_{\varphi\left(P_{2}\right)} \cong \mathbb{Z} / 5 \mathbb{Z}, L=\overline{\varphi\left(P_{1}\right) \varphi\left(P_{2}\right)}$ is not a tangent line at $\varphi\left(P_{1}\right)$, and $L$ is a tangent line at $\varphi\left(P_{2}\right)$. By Theorem 2 (1), (3) and (6), $m_{\varphi\left(P_{1}\right)}=4, m_{\varphi\left(P_{2}\right)}=23$ and $\operatorname{deg}\left(\varphi\left(\mathbb{P}^{1}\right)\right)=28$. By Theorem 2 (2), (4), and (5) the second order is equal to 4 (resp. 3,1) at each point $Q \in G_{1} \cdot P_{2} \backslash\left\{P_{2}\right\}$ (resp. at $P_{2}$, at each point $\left.Q \in G_{2} \cdot P_{1} \backslash\left\{P_{2}\right\}\right)$, and the third order is equal to 4 at $P_{2}$.

\section{Acknowledgement}

The author would like to thank Professor Satoru Fukasawa for helpful discussions and invaluable comments. The author also would like to thank the referee for his/her helpful comments.

\section{References}

[1] H. Borges and S. Fukasawa, Galois points for double-Frobenius nonclassical curves, Finite Fields Appl. 61 (2020), 101579, 8 pages.

[2] X. Faber, Finite $p$-irregular subgroups of $\mathrm{PGL}_{2}(k)$, preprint, arXiv:1112.1999.

[3] S. Fukasawa, Galois points for a plane curve in arbitrary characteristic, Geom. Dedicata 139 (2009), 211-218.

[4] S. Fukasawa, Galois points for a non-reflexive plane curve of low degree, Finite Fields Appl. 23 (2013), 69-79. 
[5] S. Fukasawa, A birational embedding of an algebraic curve into a projective plane with two Galois points, J. Algebra 511 (2018), 95-101.

[6] S. Fukasawa, Galois lines for the Artin-Schreier-Mumford curve, preprint, arXiv:2005.10073.

[7] S. Fukasawa, Algebraic curves admitting inner and outer Galois points, preprint, arXiv:2010.00815.

[8] S. Fukasawa and K. Higashine, Galois lines for the Giulietti-Korchmáros curve, Finite Fields Appl. 57 (2019), 268-275.

[9] H. Hayashi and H. Yoshihara, Galois group at each point for some self-dual curves, Geometry 2013 (2013), Article ID 369420, 6 pages.

[10] J. W. P. Hirschfeld, G. Korchmáros and F. Torres, Algebraic curves over a finite field, Princeton Univ. Press, Princeton, 2008.

[11] K. Miura, Field theory for function fields of singular plane quartic curves, Bull. Austral. Math. Soc. 62 (2000), 193-204.

[12] K. Miura, Galois points on singular plane quartic curves, J. Algebra 287 (2005), 283-293.

[13] K. Miura and H. Yoshihara, Field theory for function fields of plane quartic curves, J. Algebra 226 (2000), 283-294.

[14] H. Stichtenoth, Algebraic function fields and codes, Universitext, Springer-Verlag, Berlin, 1993.

[15] T. Takahashi, Non-smooth Galois points on a quintic curve with one singular point, Nihonkai Math. J. 16 (2005), 57-66.

[16] H. Yoshihara, Function field theory of plane curves by dual curves, J. Algebra 239 (2001), 340-355.

\author{
Kazuki Higashine \\ Graduate School of Science and Engineering \\ Yamagata University \\ Kojirakawa-machi 1-4-12 Yamagata 990-8560 Japan \\ E-mail: s182102d@st.yamagata-u.ac.jp
}

